\title{
Characterization of Protein-Protein Interactions via Static and Dynamic Light Scattering
}

\author{
Daniel Some and Sophia Kenrick \\ Wyatt Technology Corp. \\ USA
}

\section{Introduction}

Light scattering in its various flavors constitutes a label-free, non-destructive probe of macromolecular interactions in solution, providing a direct indication of the formation or dissociation of complexes by measuring changes in the average molar mass or molecular radius as a function of solution composition and time. It is a first-principles technique, thoroughly grounded in thermodynamics, permitting quantitative analysis of key properties such as stoichiometry, equilibrium association constants, and reaction rate parameters.

In the past, light scattering experiments on interacting protein solutions have been labor intensive and tedious, requiring large volumes of sample, and hence impeding widespread adoption by protein researchers. Recent advances in instrumentation and technique hold the promise for simplifying and automating measurements, as well as reducing sample requirements, thus broadening the appeal of these methods to the wider community of analytical biochemistry, biophysics, and molecular biology research. Pioneering work in automating and applying these measurements to equilibrium protein-protein interactions appeared in 2005-2006 (Attri \& Minton, 2005a, 2005b; Kameyama \& Minton, 2006). This chapter deals primarily with such automated methods.

\section{Theory of light scattering from biomacromolecules in solution}

\subsection{Static light scattering}

Static light scattering (SLS) measurements quantify the "excess Rayleigh ratio" $R$, which describes the fraction of incident light scattered by the macromolecules per unit volume of solution. Knowledge of $R$ vs. scattering angle $(\theta)$ and concentration $c$ may determine molar mass, size and self-interactions of the sample, while $R(t)$ will describe the kinetics of selfassociation or dissociation, via time-dependent changes in the average molar mass or size. Likewise, characterization of $R$ vs. the composition $([A],[B], \ldots)$ of a multi-component system, such as hetero-associating proteins, may determine the stoichiometry and equilibrium binding affinity of such a system, as well as binding or dissociation kinetics.

The basic theory of static light scattering from macromolecules in solution is available in myriad publications, including elementary textbooks dealing with physical chemistry (e.g., van Holde et al., 1998; Teraoka, 2002) or essential references on polymers (e.g., Young, 1981). We will cite from these without further reference. More rigorous publications, particularly 
those dealing with non-ideal, multi-component solutions are found in the scientific literature (e.g., Blanco et al., 2011, and references therein).

\subsubsection{Static light scattering in the ideal limit}

Macromolecules in solution are subject to correlations arising from intermolecular potentials, which in turn affect the magnitude of scattered light. However, if the particles are few and far between, and the potentials between them sufficiently short-ranged, these correlations may be ignored, leading to what is known as "the ideal limit": essentially, the particles behave like an ideal ensemble of point particles.

The simplest picture of scattering from proteins in solution invokes the ideal limit, i.e., point-like particles with no interactions, much like the more commonly known ideal gas law for pressure and temperature. In this case, the scattering from particles much smaller than the wavelength of incident light, with detectors placed in the plane perpendicular to the incident polarization, can be described by Eq. (1):

$$
R=\frac{4 \pi^{2} n_{0}^{2}}{N_{A} \lambda_{0}^{4}}\left(\frac{d n}{d c}\right)^{2} M c=K * M c
$$

In Eq. (1), $N_{A}$ represents Avogadro's number, $d n / d c$ is the protein's refractive increment, $M$ the protein's molar mass, $n_{0}$ the solvent refractive index, $\lambda_{0}$ the wavelength in vacuum, and $c$ the protein concentration in units of mass/volume. $K^{*}$ incorporates the constants $n_{0}, N_{A}, \lambda_{0}$ and $d n / d c$.

The protein refractive increment $d n / d c$ describes the change in refractive index of a solution relative to pure buffer, due to a mass/volume protein concentration $c$; this parameter may be readily measured by means of a common instrument known as a differential refractometer and is, fortuitously, nearly invariable for most proteins in standard aqueous buffers at any given wavelength $(d n / d c=0.187 \mathrm{~mL} / \mathrm{g}$ at $\lambda=660 \mathrm{~nm})$. High concentrations of excipient will affect $d n / d c$; adding for example arginine, which has a refractive index higher than that of most proteins, can even reduce $d n / d c$ to zero such that no scattering occurs!

For a solution consisting of multiple macromolecular species, e.g., monomer + oligomers or $\mathrm{A}+\mathrm{B}+\mathrm{AB}$ complex, the total light scattered is the sum of intensities scattered by each species:

$$
R=K^{*} \sum_{i} M_{i} c_{i}=K^{*} M_{w} c
$$

Here $M_{i}$ and $c_{i}$ refer to the molar mass and concentration of each species $i, M_{w}$ is the weightaveraged molar mass and $c$ the total protein concentrations. We have assumed that all species have the same refractive increment and non-ideality may be ignored.

Upon inspection of Eq. (2) it becomes clear that given knowledge of the measurement conditions (solution refractive index, scattering wavelength), sample parameters $(d n / d c)$, sample concentration (e.g., by means of a UV absorption or differential refractive index concentration detector), and excess Rayleigh ratio $R$, it is possible to determine the weightaveraged molar mass of macromolecules in the solution. If the solution is monodisperse (as is often the case in the course of chromatographic fractionation), then the molar mass of the solvated macromolecule may be determined. 
Eq. (2) contains the reason that light scattering is famously sensitive to small quantities of dust or other particulates: if the mass if the dust particle is a million times that of the protein, only one-millionth the concentration of dust particles produces the same scattering intensity as the protein.

Generalization of Eq. (2) to species with different refractive increments is obvious, but for reasons of simplicity we will assume henceforth equal $d n / d c$ for all proteins. This "ideal gas law for light scattering" is generally applicable to characterization of specific proteinprotein binding with equilibrium dissociation constants $K_{\mathrm{D}} \leq 1-10 \mu \mathrm{M}$.

Angular dependence of the scattered intensity comes into play for larger particles such as protein aggregates whose radii exceed $\sim \lambda / 50$. In the limit of transparent particles with radii below $\sim 40 \mathrm{~nm}$, this dependence is described by the Rayleigh-Ganz-Debye (RGD) equation:

$$
R(\theta)=K^{*} M c P(\theta) ; \quad P(\theta)=1-\frac{16 \pi^{2}}{3 \lambda_{0}^{2}}\left\langle r_{g} 2\right\rangle \sin ^{2}(\theta / 2)+\ldots
$$

For this reason SLS is often referred to as multi-angle light scattering (MALS). Here $\theta$ is the angle between the incident and scattered light rays within the plane perpendicular to the incident polarization, $r_{g}$ is the radius of gyration, and higher order terms in $P(\theta)$ have been ignored. For globular proteins, $r_{g}$ will be $\sim 80 \%$ the average geometrical radius. When $r_{g}<8-$ $12 \mathrm{~nm}, P(\theta) \sim 1$, angular dependence is eliminated, and the molecules are considered isotropic scatterers. Since the dimensions of most proteins and complexes are below $20 \mathrm{~nm}$, for the remainder of this chapter we will assume isotropic scattering, i.e., $P(\theta)=1$.

\subsubsection{Analysis of protein complexes via static light scattering coupled to online chromatographic separation}

Analytical size-exclusion chromatography (SEC) is often an unreliable measure of molar mass, particularly if the standards used to calibrate column elution times do not represent the sample well in terms of shape or column interactions. Because it does not need to make any assumptions regarding separation models or column calibration standards, flow-mode MALS is an invaluable extension of analytical SEC (SEC-MALS) or asymmetric-flow field flow fractionation (AF4-MALS). The analysis almost invariably occurs at concentrations well below $1 \mathrm{mg} / \mathrm{mL}$, low enough to fall squarely within the ideal limit. Figure 1 shows a typical SEC-MALS experimental layout, combining a MALS detector with concentration analysis by means of UV/Vis absorption or differential refractometry (dRI).

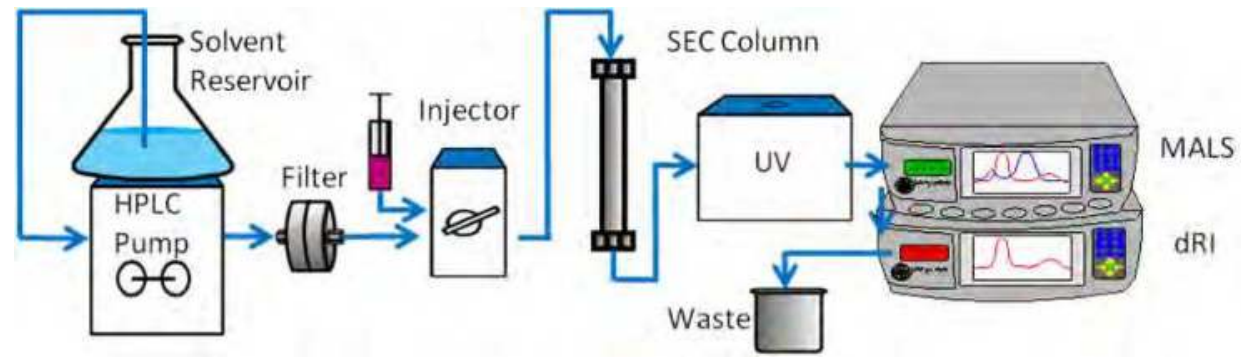

Fig. 1. SEC-MALS instrumentation. 
While normally applied to characterize polydisperse ensembles, irreversible oligomers or other tightly-bound complexes, SEC-MALS may be used to assess reversible protein interactions, especially self-association (Bajaj et al., 2007). In this approach, the excess Rayleigh ratio and concentration is measured at multiple points along an eluting peak, and these data are fit to equations representing mass conservation, mass action and ideal light scattering identical to those in Section 2.1.3. Since the ratio of monomer to oligomer in a reversibly associating system is concentration-dependent, the change in weight-average molar mass across the peak should indicate dissociation of oligomers upon dilution in the column. The measurement may be repeated at different initial sample concentrations to enhance the analysis and establish whether the dissociation kinetics are fast or slow.

While this analysis can provide a good semi-quantitative characterization of reversible association, it is subject to certain systematic errors. The analysis must assume either rapid or very slow equilibration. As the sample proceeds through the column and detectors, it dilutes continuously; if the equilibration is neither very fast nor very slow compared to the elution time, the ratio of complex to monomer will not represent equilibrium conditions. Also, band-broadening between detectors means that the concentration measured in the UV or dRI concentration detector is somewhat different than that in the MALS cell, hence systematic errors in the analysis will arise. An integrated UV-SLS cell can eliminate the latter source of error (Bajaj et. al., 2004).

The advantages of analyzing reversible complexes via SEC-MALS are low sample quantity and clean data with little noise due to particulates if the size exclusion column and HPLC system are very clean. The column will separate any dust or aggregates from the sample.

\subsubsection{Quantifying specific, reversible protein-protein binding via composition-gradient static light scattering}

The use of stop-flow injections with well-defined concentrations permits true equilibrium analysis. In some instances the kinetics of association or dissociation may be analyzed as well. Composition-gradient light scattering apparatus, described in section 3, is more generally useful than SEC-MALS for studying protein-protein interactions. This section presents the principles of this approach.

The analysis of specific, reversible protein-protein interactions in the ideal limit, via light scattering measurements from a series of compositions, has been presented concisely by Attri \& Minton (Attri \& Minton, 2005b). The equations, with a minor change in notation, include the ideal light scattering law (4), mass action (5) and conservation of mass (6), shown below assuming up to two constituent monomeric species $\mathrm{A}$ and $\mathrm{B}$ :

$$
\begin{gathered}
\frac{R}{K^{*}}=\sum_{i, j}\left(i M_{A}+j M_{B}\right)^{2}\left[A_{i} B_{j}\right] \\
K_{i j}=\frac{\left[A_{i} B_{j}\right]}{[A]^{i}[B]^{j}} \\
{[A]_{\text {total }}=\sum_{i, j} i\left[A_{i} B_{j}\right], \quad[B]_{\text {total }}=\sum_{i, j} j\left[A_{i} B_{j}\right]}
\end{gathered}
$$


$M_{A}$ and $M_{B}$ represent the molar masses of constituent monomers $\mathrm{A}$ and $\mathrm{B}$, respectively; $i$ and $j$ represent the stoichiometric numbers of $\mathrm{A}$ and $\mathrm{B}$ in the $A_{i} B_{j}$ complex, with $A_{1} B_{0}$ and $A_{0} B_{1}$ representing the monomers of $\mathrm{A}$ and $\mathrm{B} ;\left[A_{i} B_{j}\right]$ represents the molar concentration of the $A_{i} B_{j}$ complex; $[A]_{\text {total }}$ and $[B]_{\text {total }}$ represent the total molar concentration of $A$ and $B$ in solution; and $K_{i, j}$ is the equilibrium association constant relating equilibrium molar concentrations of the $A_{i} B_{j}$ complex and free monomer. Light scattering and concentration data acquired over a series of compositions-multiple concentrations, in the case of self-association of a single species, or a series of A:B composition ratios in the case of hetero-association-are fit to Eq. (4) by means of a standard least-squares nonlinear curve fitting procedure. This technique is known as composition-gradient multi-angle static light scattering, CG-MALS or CG-SLS. Beyond the usual curve fitting algorithms, there is an added complication of solving first at each composition and fitted parameter iteration, the nonlinear system of Eqs. (5) (one for each complex present in equilibrium with monomers) + Eqs. (6). Examples of the system of equations to be fit are presented in Section 4.4.1.

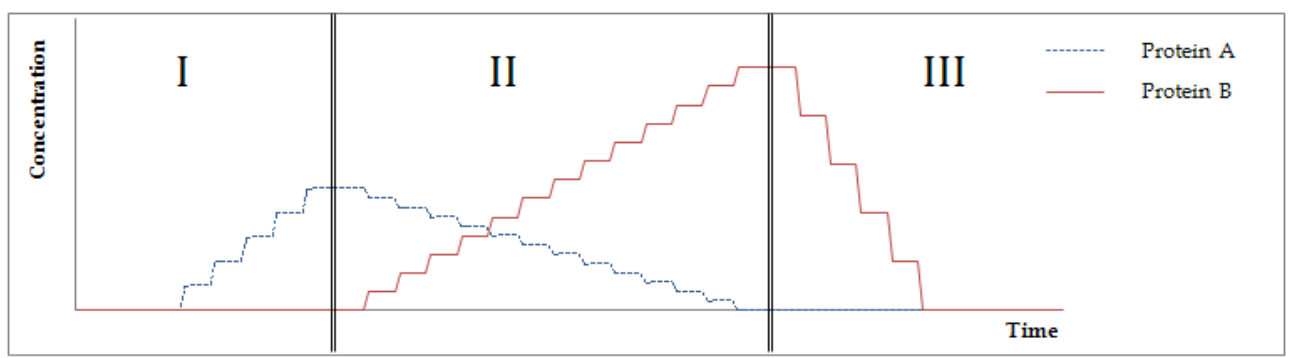

Fig. 2. Composition gradient for characterization of self- and hetero-association.

A typical composition gradient for the analysis of combined self-association and heteroassociation, shown in Figure 2, includes three segments: I and III are concentration series in A and B individually to determine any self-association, while II is a "crossover gradient" stepping through a series of $\mathrm{A}: \mathrm{B}$ composition ratios to characterize hetero-association. Figure 3a simulates LS signals for homodimer and homotrimer association, showing how the appropriate association model is well-determined by LS.
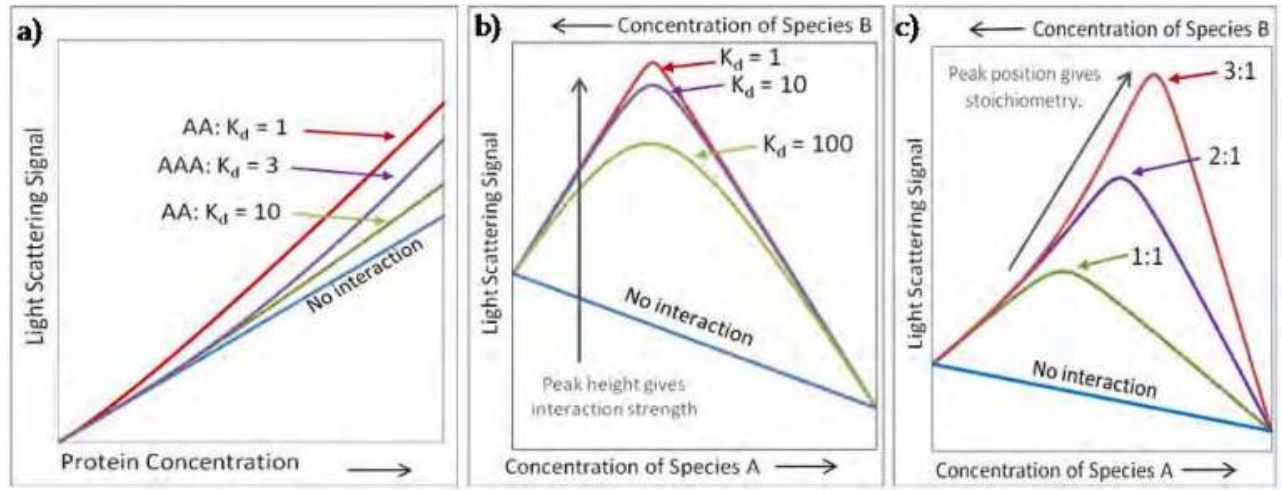

Fig. 3. Simulated CG-MALS signals. a) self association; b) and c) hetero-association. 
The crossover gradient, though perhaps not intuitive to those accustomed to sigmoidal titration curves, is in fact quite efficient for analyzing stoichiometry and binding affinity even in the presence of a complex interaction that may include simultaneous self- and hetero-association. Figure $3 b$ and Figure $3 c$ depict a qualitative interpretation of the behavior of the light scattering signal for such a gradient: the position of the peak along the composition gradient axis indicates the stoichiometric ratio of the complex, while the height and shape of the peak indicate both the binding affinity and the true stoichiometry, i.e., discrimination between the formation of 1:1 or 2:2 complexes.

\subsubsection{Non-ideal static light scattering for characterization of nonspecific protein interactions}

Nonspecific protein interactions arise from various sources such as hard-core molecular repulsion, net charge, dipoles, hydrophobic patches, van der Waals interactions, hydration forces, etc. These are generally weak in relation to specific binding and so only become important at concentrations exceeding about $1 \mathrm{mg} / \mathrm{mL}$. In contrast to site-specific lock-andkey binding, nonspecific interactions do not exhibit well-defined stoichiometry and do not generally saturate at some sufficiently high concentration. The dominant interaction may vary from attractive to repulsive or vice-versa as the concentration increases. The ill-defined, multi-sourced nature of nonspecific interactions lends itself to thermodynamic analysis in terms of small deviations from the ideal case described by Eq. (1), hence the designation "thermodynamic non-ideality." These interactions are of interest since they generally correlate to solubility, viscosity, and propensity for aggregation, and are also key to understanding crowded biomolecular environments such as the intracellular environment.

Akin to the virial expansion of the osmotic pressure as a power series in concentration, light scattering of dilute solutions may be analyzed in terms of a virial expansion, which actually uses the same pre-factors (virial coefficients $A_{2}, A_{3}$, or $B_{22}, B_{222}$, etc.) as the osmotic pressure equation though with a slightly different functional form:

$$
\frac{R}{K^{*}}=\frac{M c}{1+2 A_{2} M c+3 A_{3} M c^{2}+\ldots} \text { or } \frac{K^{*} c}{R}=\frac{1}{M}+2 A_{2} c+3 A_{3} c^{2}+\ldots
$$

In many cases a protein's nonspecific interactions may be modeled as those of hard, impenetrable spheres, albeit with an effective specific volume $v_{\text {eff }}$ different from that of the molecule's actual specific volume, resulting in a virial expansion containing only one independent parameter describing the non-ideality (Minton \& Edelhoch, 1982):

$$
\frac{R}{K^{*}}=\frac{M c}{1+8 v_{e f f} \mathcal{c}+30\left(v_{e f f} \mathcal{c}\right)^{2}+\ldots}
$$

Each virial coefficient may be expressed in terms of the effective volume. As the lowestorder correction to ideal light scattering, $A_{2}=4 v_{\text {eff }} / M$ tends to be of greatest interest for characterizing nonspecific interactions. Unscreened, long-range charge-charge interactions are not fit well by the effective hard sphere model.

An interesting and counterintuitive feature of purely (or primarily) repulsive interactions is that the LS intensity is not monotonic with concentration. Rather it initially rises as expected from the ideal LS equation, then plateaus, and eventually declines with higher concentration 
(see high-concentration case study, Section 5.5). Many non-associating proteins exhibit light scattering behavior which is fit well by the effective hard sphere assumption including scattering that eventually decreases with concentration (Fernández \& Minton, 2008). The same work described CG-SLS apparatus suitable for high concentrations.

\subsubsection{Quantifying repulsive and weakly attractive protein interactions via composition- gradient static light scattering at high concentration}

Attractive nonspecific interactions, though weak, will at high enough concentrations invariably lead to the formation of transient clusters which can be analyzed in terms of specific reversible oligomerization, rather than in terms of virial coefficients. The same is not true of repulsive interactions. Hence it is possible to segregate the repulsive interactions into the virial coefficients and treat the attractive interactions as specific self-association.

The analysis is further simplified by assuming not only that the monomers and oligomers behave as effective hard spheres but also that all species have the same effective specific volume $v_{\text {eff. }}$ An algorithm has been developed to include the effect of varying thermodynamic activity on the apparent association constants (Minton, 2007). This approach has been shown to work well for enzymes (Fernández \& Minton, 2009) as well as antibodies (Scherer et al., 2010). While not quite as rigorous, we have found that a reduced analysis based on Eq. (9) serves to reproduce the essential behavior at high protein concentration in terms of self-associating oligomers subject to an effective hard sphere repulsion:

$$
\frac{R}{K^{*}}=\frac{\sum_{i} i M c_{i}}{1+8 v_{e f f} \mathcal{c}+30\left(v_{e f f} \mathcal{c}\right)^{2}+\ldots}
$$

\subsection{Dynamic light scattering}

Rather than measuring the time-averaged intensity of scattered light, dynamic light scattering (DLS) measures the intensity fluctuations which arise from Brownian motion of the scattering molecules. The fluctuations are mathematically processed to produce an autocorrelation spectrum, which is then fit to appropriate functional forms to assess the translational diffusion constant $D_{t}$. $D_{t}$ can be related via the Stokes-Einstein equation to a characteristic dimension, the hydrodynamic radius $r_{h}$, which is just the radius of a sphere with the diffusion constant $D_{t}$. The theory of DLS is covered in myriad sources, from textbooks (e.g., Teraoka, 2002) to peer-reviewed scientific literature.

DLS has certain practical advantages over SLS. In particular, DLS enjoys a relative immunity to stray light, which permits robust measurements in small volumes with free surfaces, such as a microwell plate. On the other hand, DLS is not as sensitive as SLS and so requires higher concentrations, limiting the range of binding affinities it can measure.

\subsubsection{Analyzing protein-protein binding via composition-gradient dynamic light scattering in the ideal limit}

The same nonspecific interactions leading to thermodynamic non-ideality in SLS do affect the diffusion constant and apparent $r_{h}$ measured by DLS. In the ideal limit corresponding to a sufficiently dilute solution, this may be ignored. A solution consisting of multiple species 
$\mathrm{A}_{i} B_{j}$ will exhibit a diffusion constant which is the z-average of the diffusion constants $D_{t, i j}$ of the individual species, leading to an average hydrodynamic radius as shown in Eq. (10):

$$
\frac{1}{r_{h}^{a v g}}=\sum_{i, j} \frac{1}{r_{h, i j}} M_{i j}^{2}\left[A_{i} B_{j}\right] / \sum_{i, j} M_{i j}^{2}\left[A_{i} B_{j}\right]
$$

Upon measuring a series of concentrations or compositions like that shown in Figure 2, one can determine stoichiometry and equilibrium association constants by analyzing the apparent diffusion constants in terms of Eq. (10) in combination with Eq. (5) and Eq. (6). This technique is known as composition-gradient dynamic light scattering (CG-DLS).

Unlike the molar mass measured by static light scattering, it is not obvious or straightforward to construct the hydrodynamic radius or diffusion constant of an associating complex, given the hydrodynamic radii of its constituent species. This is especially true for stoichiometries higher than 1:1 where the geometry could vary from compact to extended, leading to significantly different diffusion constants. Compositiongradient DLS data has been shown to successfully analyze binding of globular proteins to an $\mathrm{A}_{\mathrm{i}} \mathrm{B}_{\mathrm{j}}$ complex by assuming a power law relationship (Hanlon et. al, 2010):

$$
r_{h, i j}=\left(i r_{h, A}^{\alpha}+j r_{h, B}^{\alpha}\right)^{\frac{1}{\alpha}}
$$

The best fits for different associating systems resulted in $\alpha$ values ranging from $\sim 2.8$ for homodimers and 1:1 complexes, to $\sim 2.0$ for a 2:1 stoichimetry. In this work, CG-DLS in a microwell plate reader provided important benefits, including low sample consumption and the ability to measure the same samples at multiple temperatures in order to obtain enthalpy and entropy of the interaction via van ' $t$ Hoff analysis.

\subsubsection{Analyzing nonspecific interactions via dynamic light scattering}

Nonspecific interactions give rise to thermodynamic non-ideality in DLS as well as MALS. The first-order correction to the translational diffusion constant incorporates a combination of parameters: the second osmotic virial coefficient $A_{2}\left(B_{22}\right)$, the specific volume of the molecule $v_{s p}$, and the first-order correction to the molecule's friction coefficient due to concentration $\zeta_{1}$, as presented in Eq. (12) (Teraoka, 2002).

$$
D_{t}=D_{0}\left(1+k_{D} c+\ldots\right) ; \quad k_{D}=2 A_{2}-2 v_{s p}-\zeta_{1}
$$

We can expect $v_{s p}$ to remain approximately constant for a given protein in different buffer systems, while $\zeta_{1}$ actually includes additional $A_{2}$ dependence. A measurement of $k_{D}$ for a series of monoclonal antibodies in different buffers exhibits excellent correlation with $A_{2}$ (Lehermayer et al., 2011).

The sample concentrations needed to measure $k_{D}$ are comparable to those needed to measure $A_{2}$, but the volumes can be much smaller. Hence, in order to track trends in nonspecific interactions with buffer modifications such as $\mathrm{pH}$ or ionic strength, $k_{D}$ (particularly as measured in a DLS plate reader) can be a low-volume, high-throughput surrogate for CG-MALS $A_{2}$ analysis. Unlike CG-MALS, however, currently the highconcentration behavior of CG-DLS is insufficiently understood to interpret data in the 10$200 \mathrm{mg} / \mathrm{mL}$ range. 


\section{Composition-gradient light scattering instrumentation}

\subsection{Detectors}

Light scattering instrumentation for solutions has evolved considerably in the past two decades, resulting in systems that make characterization of protein interactions via CGMALS and CG-DLS both feasible and accessible. Current top-of-the-line commercial MALS instruments provide a dynamic range covering protein solutions from tens of $\mathrm{ng} / \mathrm{mL}$ up to hundreds of $\mathrm{mg} / \mathrm{mL}$ (HELEOS, Wyatt Technology Corp.), accessing interactions with $K_{\mathrm{D}}$ from sub-nM to mM. Closed systems employing low-stray-light flow cells are important for low through moderately high concentrations, but are not suitable for the most concentrated protein samples that tend to be viscous. Easily-cleaned, microcuvette-based systems are better suited to the latter measurements.

A large selection of microcuvette-based DLS detectors is commercially available (Zetasizer series, Malvern Instruments; DynaPro series, Wyatt Technology; etc.). The lowest protein concentration range that these can analyze is on the order of $10-100 \mu \mathrm{g} / \mathrm{mL}$, accessing an interaction range from tens of $\mathrm{nM}$ to tens of $\mu \mathrm{M}$. Of particular note is the DynaPro DLS plate reader which can be integrated with standard liquid handling robotics to prepare automatically low-volume composition gradients.

Some instruments provide simultaneous SLS and DLS detection (HELEOS+QELS (flow cell or microcuvette), NanoStar (microcuvette), Wyatt Technology Corp; Zetasizer $\mu \mathrm{V}$ (flow cell or microcuvette), Malvern Instruments).

\subsection{Automated composition-gradient delivery systems}

An automated composition-delivery system for CG-MALS or CG-DLS is similar in many ways to standard stop-flow apparatus: two or more solutions are mixed by pumping via syringe pumps through a static mixer, an aliquot is delivered to the detector, and the flow stopped. The syringe pumps are operated at different flow ratios in order to obtain different compositions.

The most significant added requirement for light scattering is good in-line filtration of the solutions in order to eliminate large aggregates and particles generated by airborne dust, mechanical motion of syringes and valves, or protein films sloughing off surfaces. The pore size should be on the order of $0.1 \mu \mathrm{m}$ or less. One approach (Attri \& Minton, 2005a) is to add an in-line filter after the mixing point, illustrated in Figure 4. The key disadvantage of this

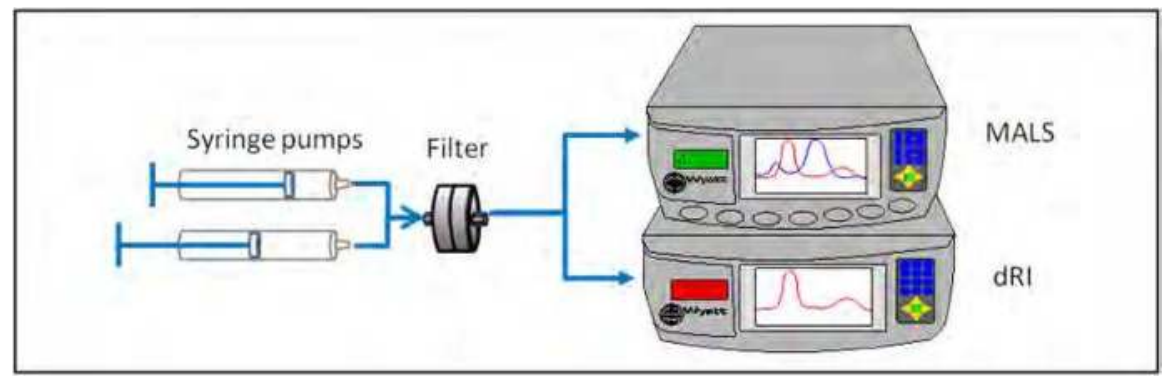

Fig. 4. Single in-line filter, parallel detectors CG-MALS setup, after Attri \& Minton 2005a. 
single-filter architecture is the changing chemical environment on the filter membrane: proteins will adhere to and release from the filter unpredictably as the environment changes. This is of particular concern in a tight-binding hetero-association analysis carried out at low protein concentrations. An in-line concentration detector is crucial.

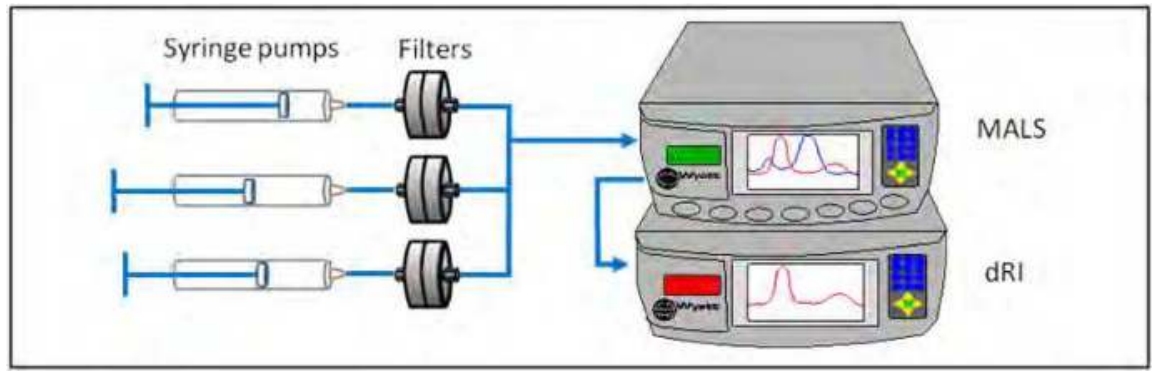

Fig. 5. Multiple in-line filters, serial detectors CG-MALS setup (Calypso, Wyatt Technology Corp.). UV or dRI concentration detector is optional.

Another approach is to flow each solution through a dedicated filter, as shown in Figure 5. In preparation for the gradient, each solution is pumped through its own filter and associated lines until saturation is reached so that, in the course of a subsequent composition gradient, well-defined compositions are produced reliably. This setup potentially eliminates the need for an in-line concentration detector if the stock protein solution concentrations are known prior to loading.

CG-MALS systems typically include light scattering and concentration detectors. The setup of Figure 5 shows a common approach, serially connected detectors, much like that of SECMALS. In order to achieve accurate correspondence between the concentration in the MALS flow cell and that in the concentration detector, both cells must be fully flushed with each composition. This can require relatively large sample volume. An alternative approach is to split the flow between the two detectors, as shown in Figure 4. Careful calibration of the flow resistance and delay between the two detectors is required to match the concentrations at the end of each injection. Additional care must be taken to ensure that laboratory temperature fluctuations, clogged capillaries or viscosity changes do not alter the split ratio between the detectors. The parallel detector configuration affords a smaller injection volume per composition.

Automation would not be complete without control and analysis software. Currently the only commercially available hardware/software package integrating syringe pump control, MALS data acquisition, and data analysis of equilibrium and kinetic macromolecular interactions is the Calypso CG-MALS system (Wyatt Technology, Santa Barbara).

\section{Practical challenges of composition-gradient light scattering}

\subsection{Sample and buffer preparation}

Due to the sensitivity of light scattering to the presence of just a few large particles, and especially in the absence of a separation step like SEC or FFF, particle-free solutions are essential in CG-MALS. Even though the composition-gradient apparatus provides in-line filtration, samples and solvents must be pre-filtered to the smallest practical pore size into 
very clean glassware or sterile, disposable containers. Solvents and buffers are generally filtered via bottle-top vacuum filters or large syringe-tip filters with pore sizes of 0.1-0.2 $\mu \mathrm{m}$. Samples should be diluted to a bit above the appropriate working concentration in filtered solvent and then filtered with a syringe-tip filter to the smallest allowable pore size, most commonly 0.02- $\mu \mathrm{m}$ (e.g., Anotop filter, Whatman). All filters should be flushed to wash out any particles prior to introducing sample or final buffers.

\subsection{Maintaining clean experimental apparatus}

Regular cleaning and maintenance of the LS detectors and sample delivery apparatus are imperative for reliable, reproducible data. As a general rule, after each experiment, the instruments should be flushed with a buffer in which the sample is soluble before changing to storage or cleaning solutions. Common detergents for removing protein and polymer residue from glass and plastic surfaces include $5 \% \mathrm{v} / \mathrm{v}$ Contrad 70 and 1\% w/v Tergazyme. Other methods useful for cleaning a dirty system include flushing with a high-salt $(0.5-1.0 \mathrm{M} \mathrm{NaCl})$ solution, $20-30 \%$ alcohol in water, or $10 \%$ nitric acid, as well as manual disassembly and cleaning. Salt and protein residues may be removed from syringes or valves by sonication.

Cleanliness of the instruments and buffers should be verified by observing noise in the MALS signals as the solutions flow through the system.

\subsection{Designing optimal methods}

The key parameters in CG-MALS experiment design are: 1) stock concentrations; 2) number and spacing of composition steps; 3 ) injected volume per step; and 4) equilibration time.

\subsubsection{Determining optimal concentrations}

Since molecular interactions are generally concentration-dependent, it is important to estimate the right concentration range that will, on the one hand, be high enough to produce a significant amount of complex, but on the other, be low enough so as not to saturate the complex leaving no free monomer. A general rule-of-thumb, assuming one has an estimate of $K_{D}$, is to prepare stock solution concentrations at 5-10x $K_{D}$. A more sophisticated approach is to perform a series of CG-MALS simulations assuming different concentrations, $K_{D}$ values and even association schemes (e.g. 1:1 or 2:1), selecting composition gradients that best discriminate between reasonably feasible models.

For self-association, a concentration gradient should include concentrations low enough that essentially no self-association occurs, as shown at the low-concentration end of Figure 3a where the no-interaction signal coincides with the associating signal. The gradient should also include concentrations high enough that at least $20-30 \%$ of the LS intensity arises from oligomers, as shown on the high-concentration end of Figure $3 a$.

For heteroassociation, the optimal $\mathrm{A}: \mathrm{B}$ stock concentration ratio is not necessarily the stoichiometric ratio, but depends on the molar masses of the molecular species. For good contrast, the total LS signal at 100\% A (right side of Figure $3 \mathrm{~b}$ ) and $100 \%$ B (left side of Figure $3 b$ ) will be nearly equal, i.e., $M_{A} c_{A} \sim M_{B} c_{B}$ where $c_{A}$ and $c_{B}$ refer to the stock concentrations of $\mathrm{A}$ and $\mathrm{B}$. This should be balanced against centering the LS peak close to the center of the crossover gradient. In particular, juggling these competing considerations can be tricky when the molar masses differ by a factor of 3 or more. If the mass ratio is large 
it may be better to perform a titration-like gradient where each injection includes a fixed concentration of the larger molecule, but varies the concentration of the smaller molecule.

Once the concentration ratio has been selected, the overall concentrations of A and B in the heteroassociation gradient should be chosen to discriminate well between $K_{D}$ values within a reasonably expected range. For example, the conditions of Figure $3 \mathrm{~b}$ discriminate well between $K_{D}$ values of 1,10 and 100 , but would not be conclusive if the actual $K_{D}$ is 0.1 or 1000 .

An initial CG-MALS analysis may yield multiple association models that fit the data well. Simulations of light scattering from new composition gradients can assist in judiciously designing a follow-on experiment to refine the analysis by eliminating some of the firstround models. Such simulations are incorporated into the Calypso software.

The concentrations required to measure nonspecific interactions characterized by the second virial coefficient $\left(A_{2}\right)$ typically range from $2-20 \mathrm{mg} / \mathrm{mL}$. For proteins, an initial estimate of $A_{2}$ may be calculated by assuming a hard sphere of the same molecular weight $(M)$ and hydrodynamic radius $(r)$. The stock concentration needed to achieve a $15 \%$ contribution to the total scattered intensity from the $A_{2}$ term (see Eq. (7)) can be calculated as per Eq. (13):

$$
c_{\text {stock }}=\frac{0.15}{2 A_{2}^{\text {sphere }} M} ; \quad A_{2}^{\text {sphere }}=\frac{16 \pi N_{A} r^{3}}{3 M^{2}}
$$

\subsubsection{Composition steps}

An adequate number of compositions must be evaluated for proper fitting of CGMALS/DLS data to an appropriate interaction model. For nonspecific interactions or simple homodimerization, at least five non-zero concentrations are recommended. Likewise, at least 5 composition steps are required for 1:1 binding. More complicated interactions forming larger numbers of species in equilibrium typically require 8-10 different compositions or more.

Sometimes the composition steps, rather than being distributed evenly across a gradient, can be focused in a specific region in order to make best use of the available sample, as shown in Figure 6.
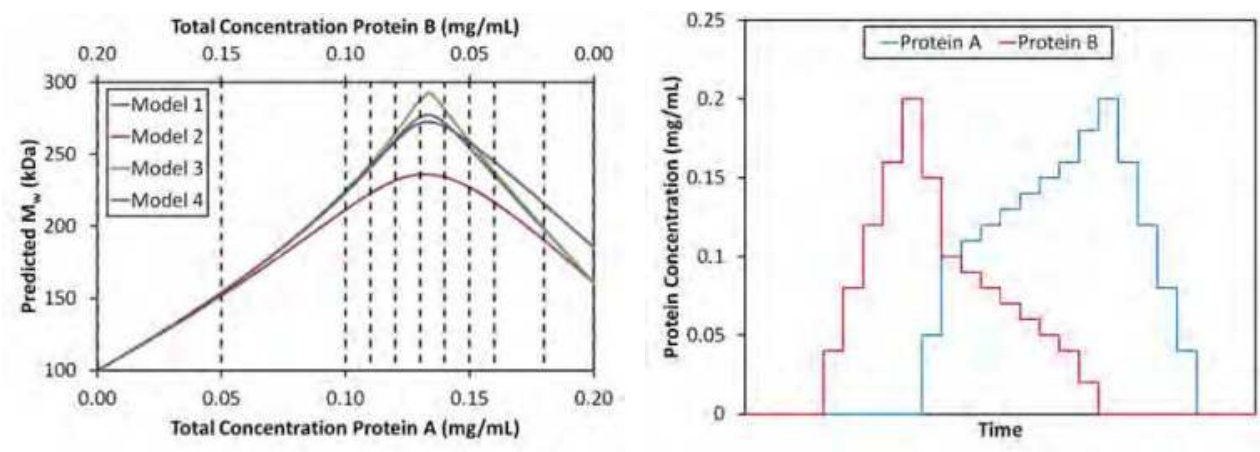

Fig. 6. Simulated interaction data for four possible interaction models (left) and corresponding CG-MALS method (right) focusing compositions around region of interest. Dashed vertical lines indicate plateau compositions. 


\subsubsection{Step volumes and equilibration time}

The volume of sample introduced to the detectors at each composition must suffice to flush out completely the previous contents of the cell. At low sample concentration, or for particularly "sticky" samples, adsorption onto surfaces (especially the in-line filter of Figure 4) may necessitate increased step volumes. The required injection size may also vary with flow rate as well as detector configuration, and so should be determined experimentally for any set of conditions. The proper step volume may be assessed by running an ascending gradient followed by a descending gradient at a series of injection volumes: as the volume increases, the signals will match more closely.

After each injection, flow is stopped and the sample given time to reach equilibrium. Often the time scale for the reaction is faster than the dead time (the time between mixing and reaching the flow cell), but when this is not the case, ample time should be allotted after each injection for equilibration.

Where sample volume is scarce or where high concentration or viscosity prevents performing stop-flow experiments, CG-MALS analyses may be performed using a microcuvette. Stock solutions for each composition are prepared in advance. The light scattering intensity from each sample is measured using a calibrated cuvette and analyzed as for a flow system. Microcuvettes must be carefully cleaned and dried between samples.

\subsection{Data analysis}

CG-MALS data analysis protocols include two distinct segments: pre-processing and model fitting. The former comprises basic steps common to many measurements: baseline subtraction, application of proportionality (calibration or conversion) factors, smoothing, and selecting the data points for analysis. Specific to multi-angle light scattering are despiking and detector selection, since the main source of noise is foreign particles that primarily affect lower scattering angles and always generate positive signals. For equilibrium analysis, data should be selected after equilibration at each step, and usually a range of data points from each composition step are averaged to provide a single value from each detector.

\subsubsection{Equilibrium models: Fitting and interpretation}

The essential parameters in a CG-MALS model are monomer molar mass $M_{A}$ and $M_{B}$; association stoichiometries $i j$; association constants $K_{A, i j}$; and incompetent (inactive) fractions $f_{\text {incomp }, A}, f_{\text {incomp }, B}$. The latter refer to protein molecules in the stock solution that are incompetent to participate in the interaction due to mutation, misfolding, aggregation, etc. In the course of fitting the data, a set of stoichiometries ij must be selected. Parameters such as monomer molar mass and incompetent fractions may be constrained to known values or floated to be optimized in the fit. Additionally, constraints may be imposed on the association constants, e.g., models of equivalent binding sites or isodesmic association confer specific relationships between the $K_{A, i j}$, as described in Table 1.

Standard iterative non-linear curve fitting algorithms, such as Levenberg-Marquardt, are implemented. For each composition, the total concentration of each monomer species is known either from precise dilution or by measurement with a UV or dRI detector. At each iteration of the free parameters, the equations for mass action and mass conservation (Eqs. 
(5) and (6)) are solved; then the light scattering is computed (Eq. (4)) and compared to the measured value, the difference thereof serving as the minimization function. The result of fitting the data to a particular model will provide association constants plus any other free parameters, as well as a measure of goodness of fit, such as $\chi^{2}$.

A broad range of useful equilibrium association models may be implemented, including any combination of self-interactions (formation of oligomers) and hetero-associations (stoichiometries of 1:1, 2:2, 1:n, etc.). Several common association models for proteins are presented in Table 1. Examples of these and more complex association schemes are discussed in Section 5.

Although useful, appropriate fitting of CG-MALS data does not require a priori knowledge of the interaction stoichiometry or system constraints. For a well-designed experiment, the best fit of the data should naturally converge on a single solution. This is illustrated in Figure 7 where incorrect models are applied to LS data for 1:1 and 1:2 interactions. In Figure 7A, which depicts a 1:2 interaction with equivalent binding sites as for antibody-antigen binding, a first guess of 1:1 interaction creates a fitted curve that does not peak at the correct stoichiometric ratio and clearly does not fit the data. Similarly, applying combined 1:1 and 1:2 stoichiometries, unconstrained for equivalent and independent binding sites, to a system with only one binding site results in the fitting algorithm eliminating the contribution from the 1:2 species (LS contribution from $\mathrm{AB}_{2}=0$ for all compositions, Figure 7B).

In many instances, the expected interaction scheme fits the data well, resulting in low $\chi^{2}$ and random residuals. Otherwise different stoichiometric models should be tested until the measured LS behavior is well matched. If the experiment design was far from optimal for the true system behavior or the interaction is particularly complex, more than one model may fit the data equally well. Several strategies may be brought to bear on selecting the most appropriate scheme, including Occam's razor (i.e., the simplest model that fits the data) and information from other techniques such as crystallographic structure or NMR analysis of binding sites. Simulation tools are useful in designing follow-up experiments to discriminate between multiple possibilities.
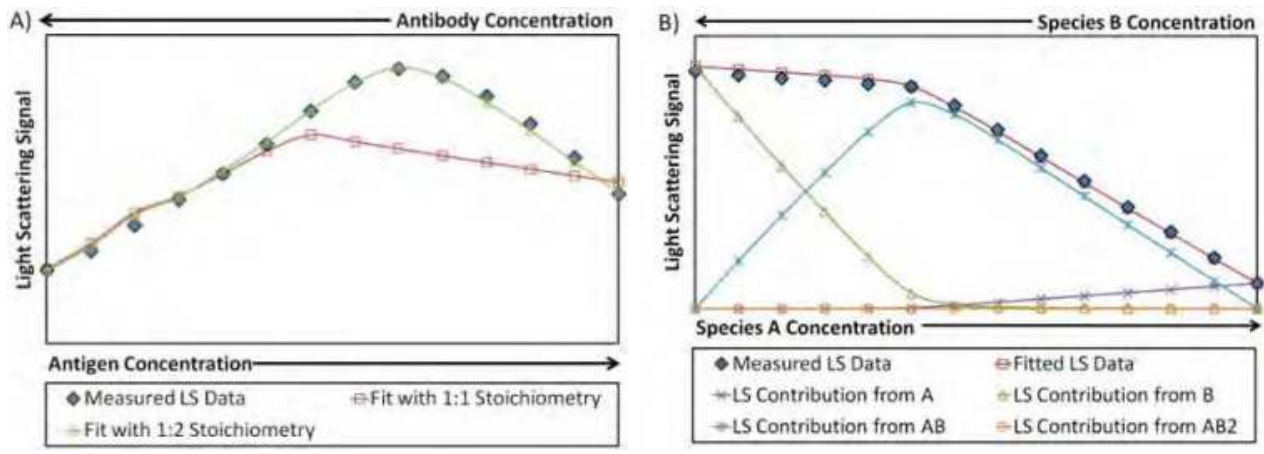

Fig. 7. Proper fitting of CG-MALS data requires the correct association model. A) Fitting of 1:2 interaction by 1:1 or 1:2 stoichiometry. B) Best fit of interaction between chymotrypsin (A) and bovine pancreatic trypsin inhibitor (B) from crossover gradient in Section 5.2.1 requires only 1:1 interaction. 


\begin{tabular}{|c|c|c|}
\hline Interaction Model & Unknowns & Governing Equations \\
\hline $\begin{array}{l}\text { Dimerization } \\
\text { (e.g., Section 5.1.1) }\end{array}$ & $K_{20}=\frac{\left[A_{2}\right]}{[A]^{2}}$ & $\begin{array}{l}R / K^{*}=M_{A}^{2}\left([A]+4 K_{20}[A]^{2}\right) \\
{[A]_{\text {total }}=[A]+2 K_{20}[A]^{2}}\end{array}$ \\
\hline $\begin{array}{l}\text { 1:1 hetero-association } \\
\text { (e.g., Section 5.2.1) }\end{array}$ & $K_{11}=\frac{[A B]}{[A][B]}$ & $\begin{array}{l}R / K^{*}=M_{A}^{2}[A]+M_{B}^{2}[B]+\left(M_{A}+M_{B}\right)^{2} K_{11}[A][B] \\
{[A]_{\text {total }}=[A]+K_{11}[A][B]} \\
{[B]_{\text {total }}=[B]+K_{11}[A][B]}\end{array}$ \\
\hline $\begin{array}{l}\text { 1:2 hetero-association, } \\
\text { equivalent binding } \\
\text { sites } \\
\text { (e.g., Section } 5.2 .2 \text { ) }\end{array}$ & $\begin{array}{c}K_{11}=\frac{[A B]}{[A][B]} \\
K_{12}=\frac{\left[A B_{2}\right]}{[A][B]^{2}}=\left(\frac{K_{11}}{2}\right)^{2}\end{array}$ & $\begin{aligned} R / K^{*} & =M_{A}^{2}[A]+M_{B}^{2}[B]+\left(M_{A}+M_{B}\right)^{2} K_{11}[A][B] \\
& +\frac{1}{4}\left(M_{A}+2 M_{B}\right)^{2} K_{11}^{2}[A][B]^{2} \\
{[A]_{\text {total }} } & =[A]+K_{11}[A][B]+\frac{1}{4} K_{11}^{2}[A][B]^{2} \\
{[B]_{\text {total }} } & =[B]+K_{11}[A][B]+\frac{1}{2} K_{11}^{2}[A][B]^{2}\end{aligned}$ \\
\hline $\begin{array}{l}\text { Isodesmic self- } \\
\text { association to } \\
n \text {-mers } \\
\text { (e.g., Section 5.1.2; see } \\
\text { Kameyama \& Minton } \\
\text { 2006) }\end{array}$ & $\begin{array}{c}K_{20}=\frac{\left[A_{2}\right]}{[A]^{2}} \\
K_{n 0}=\frac{\left[A_{n}\right]}{[A]^{n}}=K_{20}^{n-1}\end{array}$ & $\begin{array}{l}R / K^{*}=M_{A}^{2} \sum_{i=1}^{n} i^{2} K_{20}^{i-1}[A]^{i} \\
{[A]_{\text {total }}=\sum_{i=1}^{n} i K_{20}^{i-1}[A]^{i}}\end{array}$ \\
\hline
\end{tabular}

Table 1. Common equilibrium association models that can be quantified by CG-MALS.

\subsubsection{Kinetics models: Fitting and interpretation}

Reaction kinetics for reversible and irreversible associations can be observed and quantified by light scattering to provide a direct measure of association, dissociation, or aggregation via the evolution of $M_{w}$. Quantifying characteristic rate constants from CGMALS data requires knowledge of the final stoichiometry and, in the case of reversible associations, the appropriate equilibrium association constants. For example, LS data for covalent inhibition of an enzyme by an inhibitor may be fit at varying inhibitor concentrations to yield a second-order rate constant for the interaction, $k_{a}$. In the case of irreversible dissociation, the apparent first-order kinetics can be described by an exponential function, and the apparent dissociation rate constant, $k$, can be related to applicable biomolecular constants:

$$
\left(R / K^{*}\right) \propto\left[A_{2}\right]=\left[A_{2}\right]_{0} e^{-k t}
$$

More complex analyses, such as the association of two proteins into an equilibrium complex, involve solving the rate equations that govern the system of interest. The equilibrium association constant $K_{A}$ and final stoichiometry must be determined in addition to the timedependent change in light scattering. For the simplest heteroassociation $A+B \leftrightarrow A B$, Eq. (15) relates the CG-MALS data to the second order association rate constant $k_{a}=K_{\mathrm{A}} \cdot k_{\mathrm{d}}$ : 


$$
\frac{1}{K^{*}} \frac{d R}{d t}=2 M_{A} M_{B} \frac{d[A B]}{d t} ; \quad \frac{d[A B]}{d t}=k_{a}\left\{\left([A]_{\text {total }}-[A B]\right)\left([B]_{\text {total }}-[A B]\right)-\frac{[A B]}{K_{A}}\right\}
$$

\section{CG-MALS examples}

\subsection{Self-association}

\subsubsection{Dimerization of chymotrypsin}

Dimerization has been observed by CG-SLS for the enzyme $\alpha$-chymotrypsin with $\mathrm{pH}$ dependent affinity (Kameyama \& Minton, 2006; Fernández \& Minton, 2009). Figure 8 presents dependence of the reaction on ionic strength (Hanlon \& Some, 2007), closely matching results obtained via sedimentation equilibrium (Aune et al., 1971).

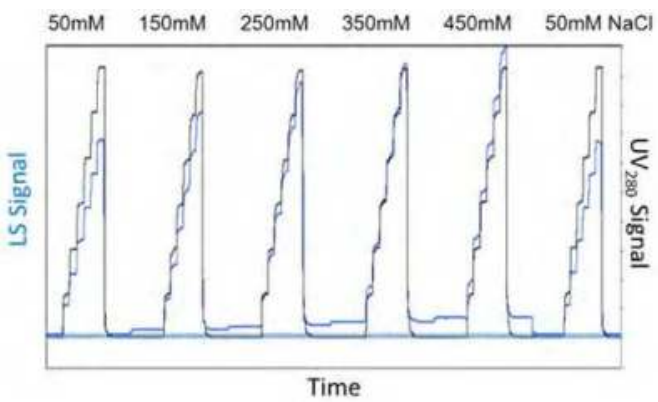

(A)

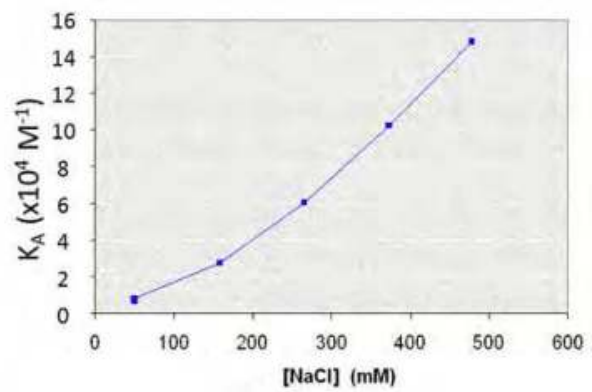

(B)

Fig. 8. Self-association of chymotrypsin forming dimers vs. ionic strength. (A) LS and UV 280 concentration data over a series of concentration gradients (B) $K_{\mathrm{A}} \mathrm{vs}$. [NaCl].

\subsubsection{Isodesmic self-association}

Some proteins tend to self assemble into chains, fibrils, or other large oligomers, such as amyloid- $\beta$ plaques in Alzheimer's disease and $\alpha$-synuclein aggregates in the Lewy bodies of Parkinson's disease. A model of isodesmic self-association, i.e., the assumption that each protein monomer binds to the growing chain with equal affinity, can often be used to describe such an interaction, especially in the early nucleation phase of the assembly.

Insulin changes its self-association state as a function of $\mathrm{pH}$ and the presence of zinc ions (Attri et al., 2010a, 2010b, and references therein). At physiological conditions in the presence of $\mathrm{Zn}^{2+}$, insulin exists as a hexamer that further associates isodesmically to higher order oligomers - dimers of hexamers (12-mers), trimers of hexamers (18-mers), etc. (Attri et al., 2010b). This interaction was studied using both static and dynamic light scattering. Based on the reported equilibrium and diffusion constants, $\mathrm{M}_{\mathrm{w}}, \mathrm{D}_{\mathrm{t}}$, and the molar composition of insulin oligomers could be reproduced (Figure 9).

In contrast, in the absence of $\mathrm{Zn}^{2+}$, insulin monomers exist in isodesmic equilibrium with dimers, trimers, and higher order complexes with $\mathrm{pH}$-dependent affinity (Figure 10). Rather than constraining the maximum oligomerization state as in Table 1, both studies considered the possibility of infinitely large oligomers. 

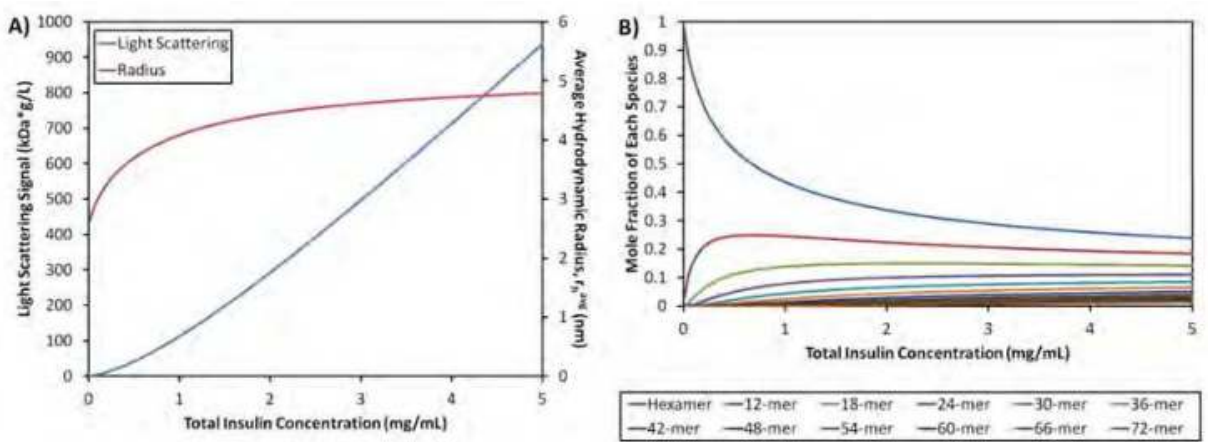

Fig. 9. Infinite self-association of insulin hexamers at neutral $\mathrm{pH}$ in the presence of $\mathrm{Zn}^{2+}$. A) LS signal and $\mathrm{r}_{\mathrm{h}}{ }^{\text {avg }}$ vs. protein concentration, calculated per $K_{\mathrm{A}}$ and $D_{t}$ in Attri et al., 2010b.

B) Calculated molar distribution of species.
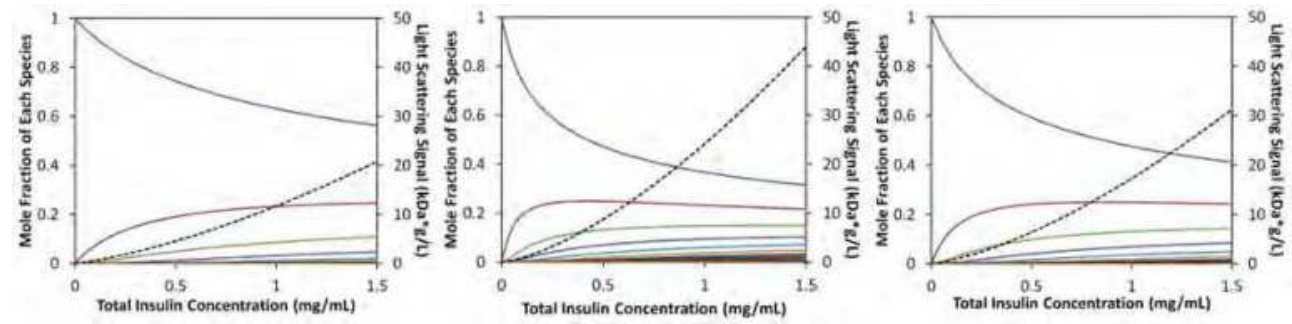

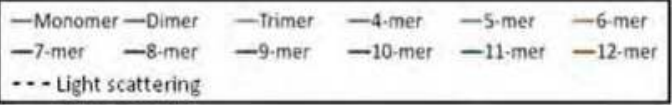

Fig. 10. Molar distribution of insulin self-association products and light scattering signal in the absence of $\mathrm{Zn}^{2+}$ at pH 3 (left), 7.2 (middle), and 8 (right), calculated per Attri et al., 2010a.

\subsection{Hetero-association}

\subsubsection{Reversible enzyme-inhibitor binding with 1:1 stoichiometry}

Following Kameyama \& Minton 2006, we characterized a standard 1:1 reversible association between $\alpha$-chymotrypsin (CT) and bovine pancreatic trypsin inhibitor (BPTI). A CG-MALS experiment consisting of self-association gradients for each binding partner CT and BPTI and a crossover hetero-association gradient was performed as per Figure 2. The selfassociation gradients yield the molecular weight for each monomer and confirm the lack of self-association for CT and BPTI at neutral $\mathrm{pH}$. Fitting the LS data in Figure $11 \mathrm{~A}$ as a function of composition (Figure 7B) results in an equilibrium dissociation $K_{\mathrm{D}}=119 \mathrm{nM}\left(K_{\mathrm{A}}=\right.$ $8.5 \times 10^{6} \mathrm{M}^{-1}$ ), consistent with measurements by other techniques (referenced in Kameyama \& Minton, 2006). The LS contribution from each species is then transformed to a concentration, giving the species distribution shown in Figure 11B. As expected for a 1:1 interaction, the plateau with the highest amount of complex formation occurs at a molar ratio of CT:BPTI $\sim 1: 1(\sim 11 \mu \mathrm{M}$ each CT and BPTI). Since the experiment was performed at concentrations $>10 x K_{D}$, nearly all available free monomer is consumed in the (CT)(BPTI) complex. This is 
evident in Figure 11B where the mole fraction of $\mathrm{CT}$ is $\sim 0$ for all compositions $[\mathrm{CT}]<[\mathrm{BPTI}]$, and the mole fraction of BPTI is $\sim 0$ for all compositions $[\mathrm{BPTI}]<[\mathrm{CT}]$.
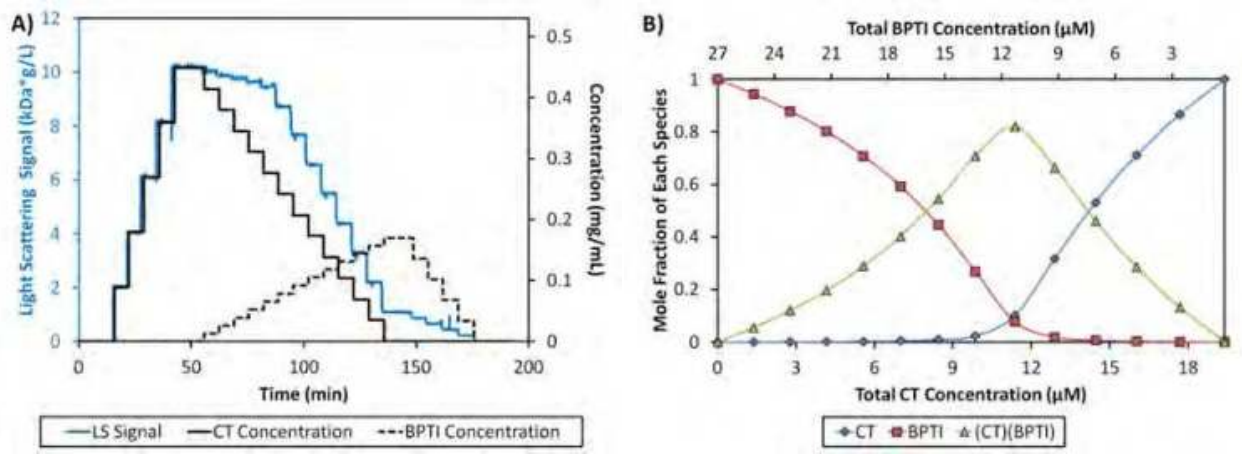

Fig. 11. CG-MALS quantifies binding of CT and BPTI.

Under acidic conditions, the affinity of CT for BPTI decreases, and CT can form reversible dimers, as in Section 5.1.1. At pH 4.4, the $K_{\mathrm{D}}$ for the association of CT and BPTI is of the same order as for the dimerization of $\mathrm{CT}-K_{\mathrm{D}}=10 \mu \mathrm{M}$ and $50 \mu \mathrm{M}$ respectively (Kameyama \& Minton, 2006). Based on these results, we can simulate the expected LS signals for simultaneous self and hetero-associations (Figure 12). Discrimination between 1:1 binding only, and self + heteroassociation, is readily evident where $[\mathrm{CT}]>[\mathrm{BPTI}]$ (Figure 12A). Despite the additional self-association, the fraction of hetero-association product still peaks at a molar ratio of CT:BPTI $\sim 1: 1$ (Figure 12B).
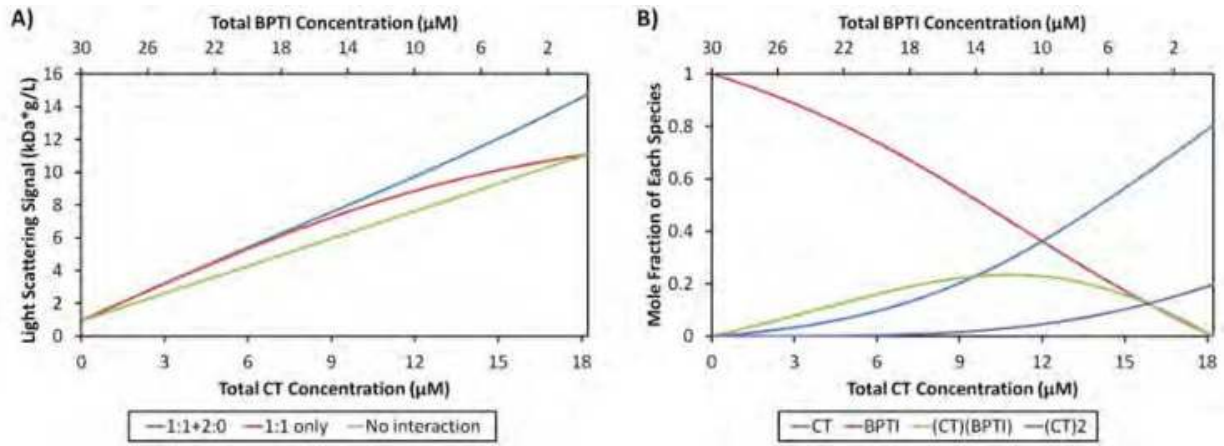

Fig. 12. CT-BPTI interaction at $\mathrm{pH}$ 4.4. A) Predicted LS for simultaneous CT self-association and CT-BPTI hetero-association (blue) compared to CT-BPTI hetero-association alone with $K_{\mathrm{D}}=10 \mu \mathrm{M}$ (red) or no interaction (green). B) Molar distribution of species for simultaneous self- and hetero-association model, based on Kameyama \& Minton, 2006.

\subsubsection{Antibody-antigen binding with 1:2 stoichiometry, two equivalent binding sites}

The power of CG-MALS lies in its ability to identify multiple stoichiometries in solution. For example, a single multivalent receptor $A$ may bind multiple protein ligands $B$, leading to the simultaneous presence of $A B, A_{2}, A_{3}$, etc. The increasing prevalence of therapeutic 
antibodies brings this type of multivalent binding to the forefront of biotechnology. Moreover, CG-MALS is able to characterize this type of interaction with affinities as low as $K_{\mathrm{D}} \sim 0.1 \mathrm{nM}$, typical of antibody-antigen interactions. Our antibody-antigen binding data (Figure 13) indicates the presence of four species in solution: free antibody $(\mathrm{Ab})$, free antigen $(\mathrm{Ag})$, the 1:1 complex $(\mathrm{Ab})(\mathrm{Ag})$, and the 1:2 complex $(\mathrm{Ab})(\mathrm{Ag})_{2}$. The CG-MALS $\mathrm{K}_{\mathrm{D}}$ value of $10 \mathrm{nM}$ agrees well with the literature value.
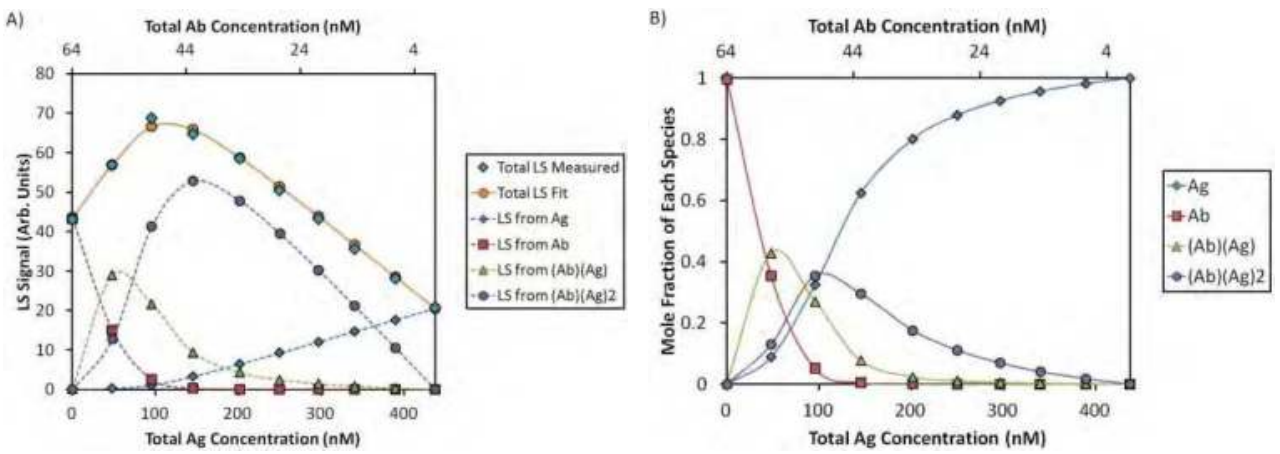

Fig. 13. Light scattering (A) and composition (B) distributions for crossover gradient between an antibody and monovalent antigen, $K_{\mathrm{D}} \sim 10 \mathrm{nM}$.

Conversely, Some et al. (2008a) found that CG-MALS data for a dimeric Fc $\gamma$ receptor (Fc $\gamma R$ ) binding to the Fc of a recombinant human $\mathrm{Ab}$ (rhumAb), shown in Figure 14, is only fit well by a model assuming two equivalent binding sites on each FcyR dimer (B) for rhumAb (A), producing equilibrium between monomers ( $A$ and $B$ ), $A B$, and $A_{2} B$ (Figure 14C). CG-MALS data do not support other binding models, including $1 \mathrm{~A}: 1 \mathrm{~B}$ association alone (Figure 14A) and $1 \mathrm{~A}: 2 \mathrm{~B}$ with equivalent binding sites (Figure 14B). The calculated single-site affinity of $50 \mathrm{nM}$ agrees closely with surface plasmon resonance (SPR) analysis.

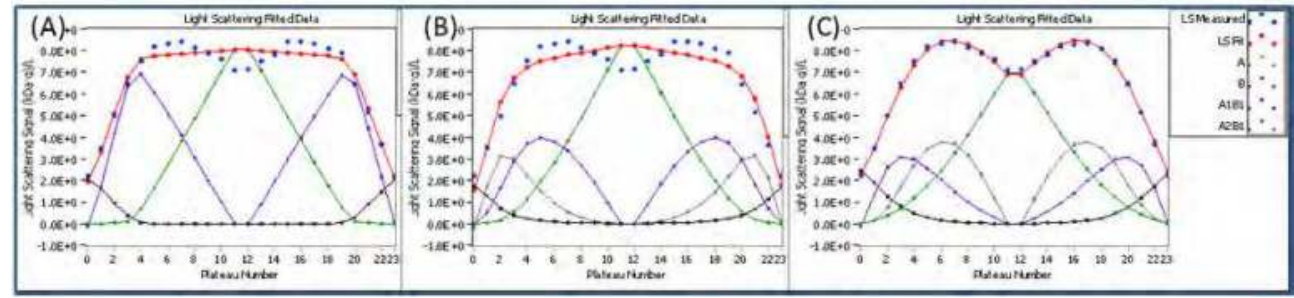

Fig. 14. Best fits (red lines) of measured CG-MALS data (blue circles) to different association

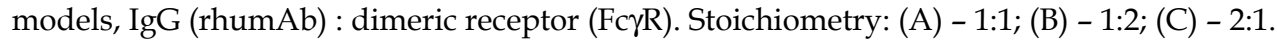
Only the $\{2 \mathrm{mAb}$ per receptor $\}$ model fits the data.

\subsubsection{Association of multivalent protein complexes}

Combinations of multivalent binding partners can lead to the formation of metacomplexes in solution that are not identified by other techniques. As a homo-tetramer, streptavidin (SA) is composed of four identical binding sites capable of binding either of two Fab domains of an anti-streptavidin IgG. As we have observed, the combination of multivalent 
proteins enables higher-order stoichiometries to present themselves in solution, including multiple IgG molecules binding a single SA molecule and self-assemblies of IgG-SA complexes (Figure 15). Indeed, the LS signal measured for such a system by CG-MALS is nearly twice the value expected for a simple 1:2 association (Figure 15). Careful analysis of the data indicates that the solution is best described as 1:1 (IgG)(SA) complexes that selfassociate (Figure 16). The infinite self-association (ISA) model employed here assumes that each base unit-(IgG)(SA) complex - assembles with other base units with the same affinity; however, this may differ from the binding-site affinity $\left(K_{D}\right)$ for a single IgG-SA interaction. The binding-site $K_{\mathrm{D}}$ for one SA molecule binding one IgG was determined as $22 \mathrm{nM}$, while these $1: 1$ base units assemble with an average affinity $K_{\mathrm{D}}=50 \mathrm{nM}$.
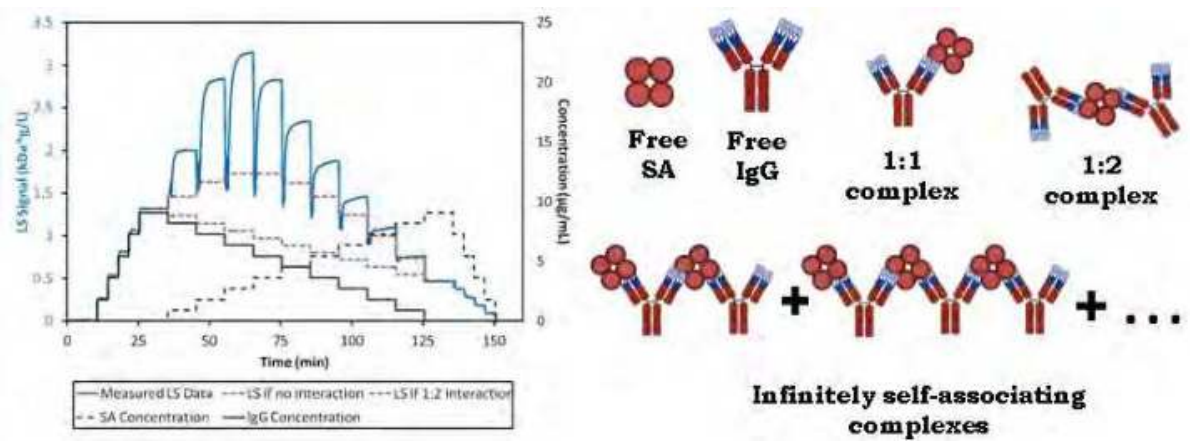

Fig. 15. Light scattering and concentration data for association of SA and anti-SA IgG. Theoretical LS plateaus are indicated for the case of no IgG-SA interaction and a 1:2 equivalent binding site model (Section 5.2.2). Additional stoichiometries that contribute to the measured LS signal, including infinitely self-associating 1:1 complexes, are shown.
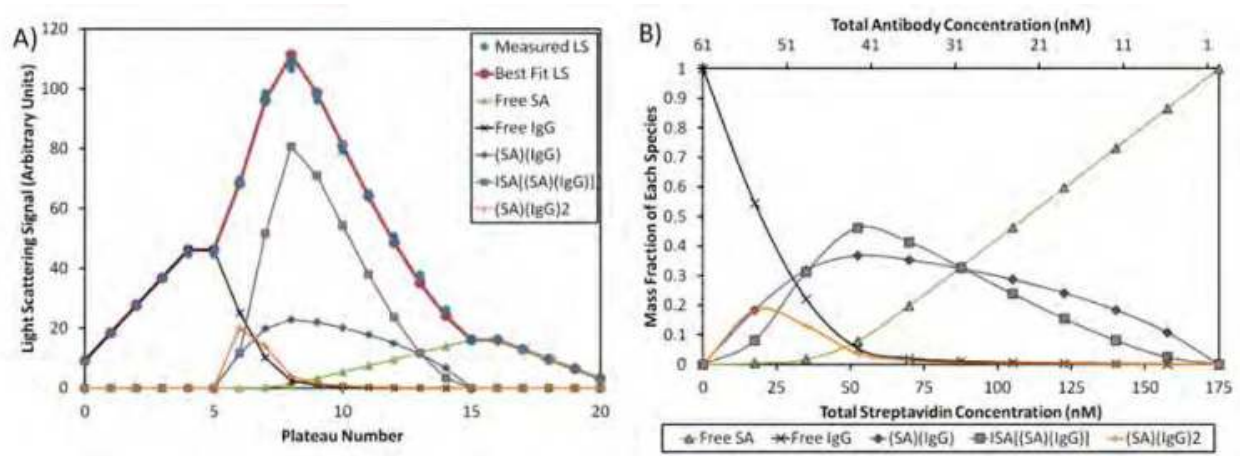

Fig. 16. A) Best fit of LS data for SA + anti-SA IgG includes infinite self assembly (ISA) of 1:1 metacomplexes B) Concentration distribution for hetero-association plateaus (\#5-15).

\subsection{Dissociation kinetics induced by a small molecule inhibitor}

Although other techniques, such as SPR and FRET-based methods, are capable of quantifying association and dissociation kinetics, many require modification of the protein of interest, i.e., immobilization in the case of SPR and labeling with fluorescent tags for 
FRET. In contrast, CG-MALS enables real-time observation of reaction kinetics in solution without protein modification. For example, chymotrypsin self-association at low $\mathrm{pH}$ is inhibited by the small molecule 4-(2-aminoethyl) benzenesulfonyl fluoride (AEBSF). When introduced to a chymotrypsin solution, AEBSF covalently binds the monomer active site and prevents dimerization. Varying concentrations of AEBSF were mixed with a constant stock solution of chymotrypsin, and the resulting dissociation kinetics quantified with a model of an irreversible dissociation (Some \& Hanlon, 2010). For each composition, the solution was allowed to react for $>1 \mathrm{hr}$ while observing the decrease in weight-average molar mass of the solution. The characteristic reaction time $\tau(1 / k$ in Eq. (14)) varies inversely with the AEBSF concentration, consistent with the rate models defined for the system (Figure 17), indicating a rate constant of $0.064 \mathrm{M}^{-1} \mathrm{~s}^{-1}$.
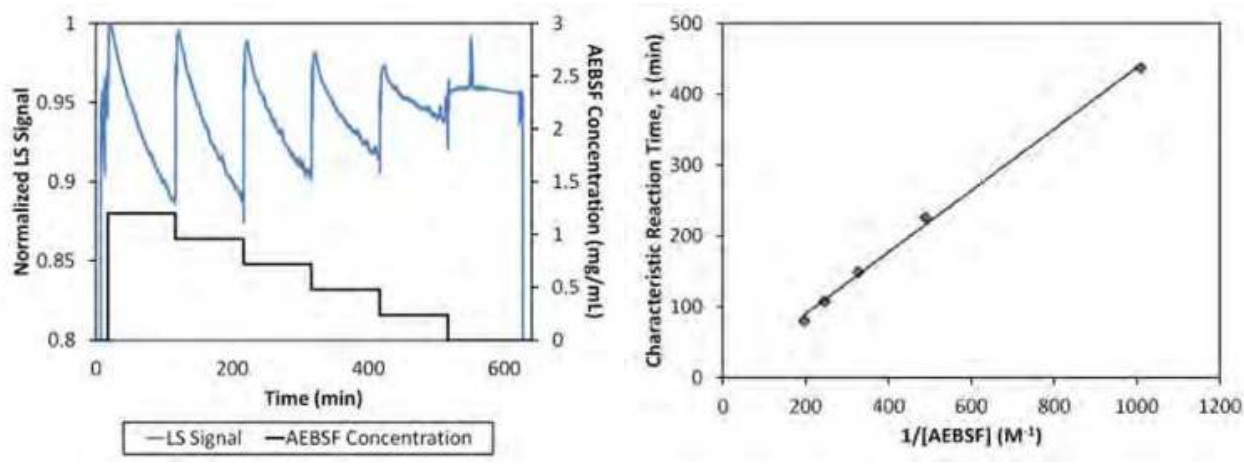

Fig. 17. Decrease in LS signal (left) and change in characteristic reaction time (right)

corresponding to irreversible dissociation of chymotrypsin dimers in the presence of AEBSF.

\subsection{Nonspecific interactions of non-self-associating proteins}

\subsubsection{Nonspecific self-interactions}

As discussed in Section 2.1.4, all macromolecules at high concentrations exhibit some degree of nonspecific interactions, quantified by the second virial coefficient, $A_{2}$. This property is of particular interest in the development of pharmaceutical formulations where $A_{2}$ is one metric for the stability of a formulation and the propensity of biomolecular therapeutics to aggregate in solution. Formulations that may appear stable at moderate concentrations $(\sim 10$ $\mathrm{mg} / \mathrm{mL}$ or less) may indeed form self-association products at relevant formulation concentrations of $100 \mathrm{mg} / \mathrm{mL}$ or more (see Section 5.5). For a well-formulated protein, however, repulsive interactions should dominate for all concentrations of interest. BSA, for example, exhibits nonspecific repulsion even at $100 \mathrm{mg} / \mathrm{mL}$ in PBS, as shown in Fig. 18. Long-range interactions are well-screened in this buffer, resulting in an $A_{2}=1.0 \times 10^{-4}$ $\mathrm{mol}^{*} \mathrm{~mL} / \mathrm{g}^{2}$, consistent with a hard-sphere of radius $3.5 \mathrm{~nm}$ and $\mathrm{M}_{\mathrm{w}}=67 \mathrm{kDa}$.

\subsubsection{Nonspecific attraction quantified by the cross-virial coefficient}

Carrier proteins, drug delivery vehicles, and other polymers attract their biomolecular targets via nonspecific interactions (e.g., Dong et al., 2011) which cannot be described by an equilibrium association constant. A virial expansion may be employed to quantify 
nonspecific attraction or repulsion between molecules of the same species or different species. In the example below, the net negative charge of BSA, in PBS with $50 \mathrm{mM} \mathrm{NaCl}$ at $\mathrm{pH} 7$, yields repulsion between BSA molecules. Lysozyme exhibits a slight positive charge with a net self-attraction as evidenced by the negative $\mathrm{A}_{2}$. The charge-mediated attraction between BSA and lysozyme molecules is evident in Figure 19 as the increase in LS when BSA and lysozyme are mixed together. The data are best fit by a model of nonspecific attraction, quantified by the cross-virial coefficient $\mathrm{A}_{11}$. The results can be normalized to a unitless value as per Sahin et al., 2010: $a_{2}=\left(A_{2}^{\text {meas }}-A_{2}^{\text {exc }}\right) / A_{2}^{\text {exc }}$.

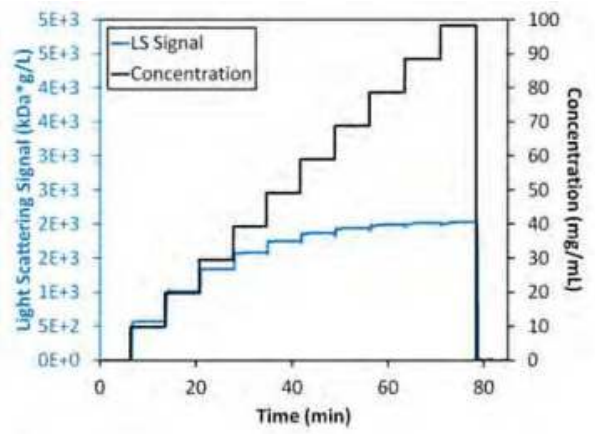

(A)

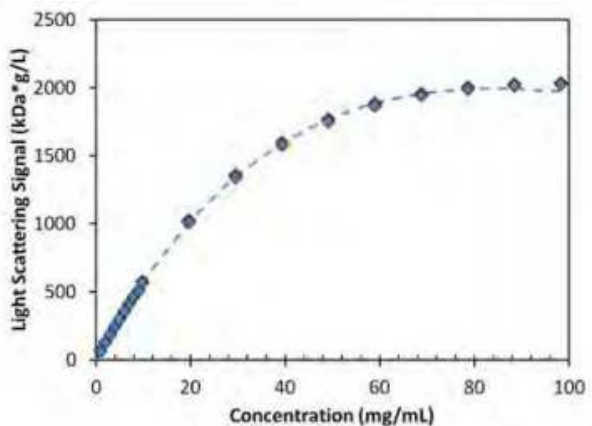

(B)

Fig. 18. BSA behaves as an effective hard sphere with $\mathrm{A}_{2}=1.0 \times 10^{-4} \mathrm{~mol}^{*} \mathrm{~mL} / \mathrm{g}^{2}$ for all concentrations studied. (A) CG-MALS data (B) fit to effective hard sphere model.

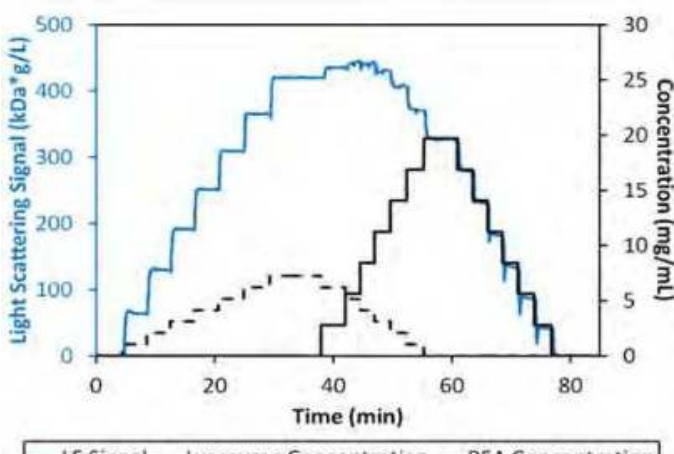

\begin{tabular}{|cc|}
\hline \multicolumn{3}{|c|}{ BSA self-virial coefficient } \\
$A_{2}\left(\mathrm{~mol}^{*} \mathrm{~mL} / \mathrm{g}^{2}\right)$ & $+1.1 \times 10^{-4}$ \\
$a_{2}$ & +0.2 \\
\hline Lysozyme self-virial coefficient \\
$A_{2}\left(\mathrm{~mol}^{*} \mathrm{~mL} / \mathrm{g}^{2}\right)$ & $-3.6 \times 10^{-4}$ \\
$a_{2}$ & -2.1 \\
\hline \multicolumn{3}{|c|}{ Cross-viral coefficient } \\
$A_{11}\left(\mathrm{~mol}^{*} \mathrm{~mL} / \mathrm{g}^{2}\right)$ & $-4.0 \times 10^{-4}$ \\
& -2.7 \\
\hline
\end{tabular}

LS Signal - Lysozyme Concentration - -BSA Concentration

Fig. 19. Determination of self-and cross-virial coefficients for nonspecific interactions in BSA-lysozyme solution. Normalized virial coefficients are also presented. 


\subsection{Interactions of monoclonal antibodies formulated at high concentration}

Recently, CG-MALS was applied to investigate interactions between IgG1 monoclonal antibodies at concentrations up to $\sim 300 \mathrm{mg} / \mathrm{mL}$ (Scherer et al., 2010). Although the two mAbs studied here were identical except for the CDR sequence, their self-association properties were remarkably different. MAb2 forms dimers with $K_{\mathrm{A}} \leq \sim 10^{3} \mathrm{M}^{-1}\left(K_{\mathrm{D}} \geq \sim 1 \mathrm{mM}\right)$, whereas mAb1 associates into dimers with $K_{\mathrm{A}} \sim 10^{3-10^{4}} \mathrm{M}^{-1}\left(K_{\mathrm{D}} \sim 0.1-1 \mathrm{mM}\right)$ and appears to further associate into higher order oligomers of stoichiometry 4-6. The dependence of association properties on ionic strength also differs dramatically between mAb1 and mAb2: while the affinity of the mAb2 homodimer increases with [ $\mathrm{NaCl}]$, that of $\mathrm{mAb} 1$ homodimers is essentially constant. Most significantly, the higher oligomer order of mAb1 decreases from 6 to 4 as $[\mathrm{NaCl}]$ increased from 40 to $600 \mathrm{mM}$.

Based on these results, we reproduce in Figure 20 the relative LS signal for mAbs1 and 2 and the fraction of each oligomer present in solution. Each calculation includes the appropriate correction for non-specific repulsion using $v_{\text {eff }}=1.8 \mathrm{~cm}^{3} / \mathrm{g}\left(r_{\text {eff }}=4.6 \mathrm{~nm}\right)$ for $\mathrm{mAb} 1$ and $v_{\text {eff }}=$ $1.4 \mathrm{~cm}^{3} / \mathrm{g}\left(r_{\text {eff }}=4.3 \mathrm{~nm}\right)$ for $\mathrm{mAb} 2$ (Scherer et al., 2010). Although the antibody molecules continue to self-associate into higher molecular weight species, the LS signal is not monotonically increasing, as would be expected from ideal scattering (Eq. (1)); instead, the LS intensity reaches a maximum at $\sim 100 \mathrm{mg} / \mathrm{mL}$ (Figure 20A). Only by accounting for both nonspecific repulsion and specific oligomerization can the light scattering data be fully described for these high-concentration solutions.
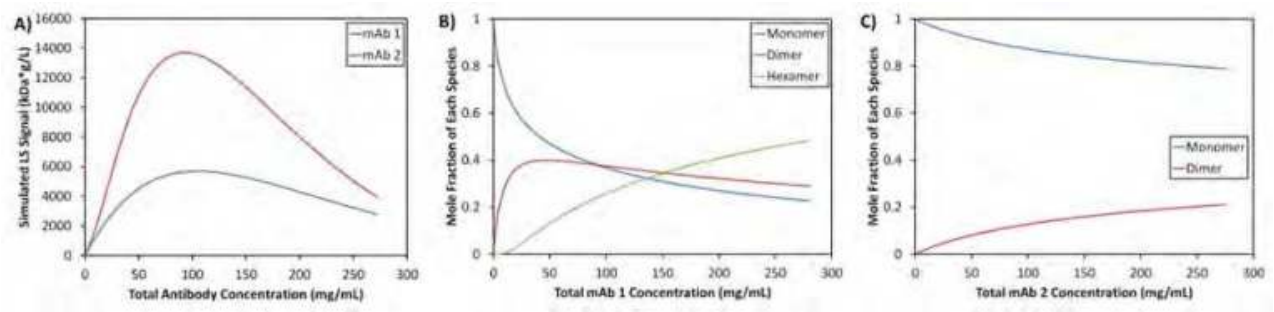

Fig. 20. A) LS signals for mAbs 1 and 2 in buffer containing $75 \mathrm{mM} \mathrm{NaCl}$, calculated to represent results of Scherer et al., 2010. B) and C) Corresponding distribution of oligomers.

\section{Conclusion}

The power of light scattering, CG-MALS and CG-DLS, for investigating protein interactions lies in their great versatility. These techniques quantify a wide range of protein-protein phenomena in solution and without labeling. Both equilibrium and kinetics may be addressed directly since light scattering provides, from first principles, the molar mass and size of complexes, rather than an indirect indicator such as fluorescence. Hence light scattering is particularly well suited to analyzing higher-order complexes, multiple stoichiometries, and simultaneous self- and hetero-association. The fundamental thermodynamic nature of static light scattering provides a critical window into interactions at high concentration. The development of automation and advanced instrumentation 
suggests that common use of CG-MALS and CG-DLS is feasible, and hence these are important additions to the protein scientist's toolbox.

\section{Acknowledgement}

The authors would like to thank Allen P. Minton for many helpful discussions and collaboration in the development of automated CG-MALS; Shawn Cao (Amgen), Joey Pollastrini (Amgen), and Jihong Yang (Genentech) for contributing antibody samples; and the entire team at Wyatt Technology Corp. We are also indebted to the many early adopters of the Calypso CG-MALS system for their support and sharing samples.

\section{References}

Attri, A.K., Fernández, C., \& Minton, A.P. (2010a). pH-dependent self-association of zincfree insulin characterized by concentration-gradient static light scattering. Biophysical Chemistry. Vol. 148, No. 1-3, (May 2010), pp. 28-33, ISSN 0301-4622

Attri, A.K., Fernández, C., \& Minton, A.P. (2010b). Self-association of Zn-insulin at neutral $\mathrm{pH}$ : investigation by concentration-gradient static and dynamic light scattering. Biophyisical Chemistry. Vol. 148, No. 1-3, (May 2010), pp. 23-27, ISSN 0304-4622

Attri, A.K. \& Minton, A.P. (2005a). New Methods for Measuring Macromolecular Interactions in Solution via Static Light Scattering: Basic Methodology and Application to Nonassociating and Self-Associating Proteins. Anal. Biochem. Vol.337, No.1, (February 2005), pp. 103-110, ISSN 0003-2967

Attri, A.K. \& Minton, A.P. (2005b). Composition Gradient Static Light Scattering (CG-SLS): A New Technique for Rapid Detection and Quantitative Characterization of Reversible Macromolecular Hetero-Associations in Solution. Anal. Biochem. Vol.337, No.1, (November 2005), pp. 103-110, ISSN 0003-2967

Aune, K.C., Goldsmith, L.C., Timasheff, S.N. (1971) Dimerization of alpha-Chymotrypsin. II. Ionic Strength and Temperature Dependence. Biochemistry Vol.10, No.9 (April 1971), pp. 1617-21, ISSN 0006-2960

Bajaj, H., Sharma, V.K., \& Kalonia, D. (2004) Determination of Second Virial Coefficient of Proteins Using a Dual-Detector Cell for Simultaneous Measurement of Scattered Light Intensity and Concentration in SEC-HPLC. Biophys. J. Vol.87, No.6, (December 2004), pp. 4048-55, ISSN 0006-3495

Bajaj, H., Sharma, V.K., \& Kalonia, D. (2007) A High-Throughput Method for Detection of Protein Self-Association and Second Virial Coefficient Using Size-Exclusion Chromatography Through Simultaneous Measurement of Concentration and Scattered Light Intensity. Pharmaceutical Research Vol.24, No.11, (November 2007), pp. 2071-83, ISSN 0724-8741

Blanco, M.A., Sahin, E., Li, Y., \& Roberts, C.J. (2011) Reexamining Protein-Protein and Protein-Solvent Interactions from Kirkwood-Buff Analysis of Light Scattering in Multi-Component Solutions. J. Chem. Phys. Vol.134, No.22, (June 2011) pp. 2251031 12, ISSN 0021-9606 
Fernández, C. \& Minton, A.P. (2008) Automated Measurement of the Static Light Scattering of Macromolecular Solutions over a Broad Range of Concentrations. Anal. Biochem. Vol.381, No.2, (Oct. 2008) pp. 254-7, ISSN 0003-2967

Fernández, C. \& Minton, A.P. (2009) Static Light Scattering from Concentrated Protein Solutions II: Experimental Test of Theory for Protein Mixtures and Weakly SelfAssociating Proteins. Biophys. J. Vol.96, No.5, (March 2009) pp. 1992-8, ISSN 00063495

Hanlon, A.D., Larkin, M.I., \& Reddick, R.M. (2010) Free-Solution, Label-Free Protein-Protein Interactions Characterized by Dynamic Light Scattering. Biophys. J. Vol.98, No.2, (Jan 2010), p. 297-304, ISSN 0006-3495

Hanlon, A.D., and Some, D. (2007). CG-MALS for Characterization of Protein Self Association and Inhibition Kinetics. International Light Scattering Colloquium 2007, Santa Barbara, October 2007

Kameyama, K. \& Minton, A.P. (2006) Rapid Quantitative Characterization of Protein Interactions by Composition Gradient Static Light Scattering. Biophys. J. Vol.90, No.6, (March 2006), pp. 2164-9, ISSN 0006-3495

Lehermayer, C., Mahler, H.-C., Mäder, K., \& Fischer, S. (2011) Assessment of Net Charge and Protein-Protein Interactions of Different Monoclonal Antibodies. J. Pharm. Sci. Vol.100, No.7, (July 2011), pp. 2551-62, ISSN 0022-3549

Minton, A.P. (2007) Static Light Scattering from Concentrated Protein Solutions, I: General Theory for Protein Mixtures and Application to Self-Associating Proteins. Biophys. J. Vol.93, No.4, (August 2007), pp. 1321-1328, ISSN 0006-3495

Minton, A.P. and Edelhoch H. (1982) Light Scattering of Bovine Serum Albumin Solutions: Extension of the Hard Particle Model to Allow for Electrostatic Repulsion. Biopolymers. Vol.21, No.2, (February 1982), pp. 451-458, ISSN 00063525

Scherer, T.M., Liu, J., Shire, S.J. \& Minton, A.P. (2010) Intermolecular Interactions of IgG1 Monoclonal Antibodies at High Concentrations Characterized by Light Scattering. J. Phys. Chem. B. Vol.114, No.40, (October 2010), pp. 12948-57, ISSN 1089-5647

Sahin, E., Grillo, A.O., Perkins, M.D., Roberts, C.J. Comparative Effects of $\mathrm{pH}$ and Ionic Strength on Protein-Protein Interactions, Unfolding, and Aggregation for AgG1 Antibodies. J. Pharm. Sci. Vol.99, No.12, (December 2010), pp.4830-48, ISSN 15206017

Some, D., Berges, A., Hitchner, E., \& Yang, J. (2008a). CG-MALS Characterization of Antibody-Antigen Interactions. International Light Scattering Colloquium 2008, Santa Barbara, October 2008

Some, D. \& Hanlon, A. (2010). Characterizing Protein-Protein Interactions via Static Light Scattering: Inhibition Kinetics And Dissociation. American Biotechnology Laboratory. Vol. 28, No. 1 (January/February 2010), pp. 9-12, ISSN 0749-3223

Some, D., Hanlon, A., \& Sockolov, K. (2008b). Characterizing Protein-Protein Interactions via Static Light Scattering: Reversible Hetero-Association. American Biotechnology Laboratory. Vol. 26, No. 4 (March 2008), pp. 18-19, ISSN 0749-3223

Teraoka, I. (2002) Polymer Solutions: An Introduction to Physical Properties, John Wiley \& Sons, Inc. ISBN 0-471-38929-3, New York, NY, USA 
van Holde, E.; Johnson, W.C. \& Ho, P. S. (1998). Principles of Physical Biochemistry, Prentice Hall, ISBN 0-13-720459-0, Upper Saddle River, NJ, USA

Young, R.J. (1981). Introduction to Polymers, Chapman and Hall, ISBN 0-412-22170-5, London, UK 


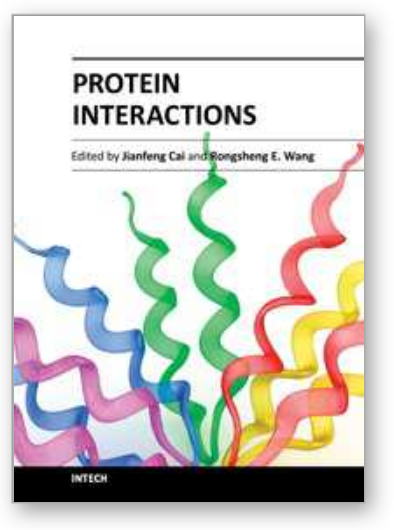

\author{
Protein Interactions \\ Edited by Dr. Jianfeng Cai
}

ISBN 978-953-51-0244-1

Hard cover, 464 pages

Publisher InTech

Published online 16, March, 2012

Published in print edition March, 2012

Protein interactions, which include interactions between proteins and other biomolecules, are essential to all aspects of biological processes, such as cell growth, differentiation, and apoptosis. Therefore, investigation and modulation of protein interactions are of significance as it not only reveals the mechanism governing cellular activity, but also leads to potential agents for the treatment of various diseases. The objective of this book is to highlight some of the latest approaches in the study of protein interactions, including modulation of protein interactions, development of analytical techniques, etc. Collectively they demonstrate the importance and the possibility for the further investigation and modulation of protein interactions as technology is evolving.

\title{
How to reference
}

In order to correctly reference this scholarly work, feel free to copy and paste the following:

Daniel Some and Sophia Kenrick (2012). Characterization of Protein-Protein Interactions via Static and Dynamic Light Scattering, Protein Interactions, Dr. Jianfeng Cai (Ed.), ISBN: 978-953-51-0244-1, InTech, Available from: http://www.intechopen.com/books/protein-interactions/characterization-of-protein-proteininteractions-via-static-and-dynamic-light-scattering

\section{INTECH}

open science | open minds

\section{InTech Europe}

University Campus STeP Ri Slavka Krautzeka 83/A 51000 Rijeka, Croatia Phone: +385 (51) 770447

Fax: +385 (51) 686166 www.intechopen.com

\section{InTech China}

Unit 405, Office Block, Hotel Equatorial Shanghai No.65, Yan An Road (West), Shanghai, 200040, China 中国上海市延安西路65号上海国际贵都大饭店办公楼 405 单元 Phone: +86-21-62489820

Fax: +86-21-62489821 
(C) 2012 The Author(s). Licensee IntechOpen. This is an open access article distributed under the terms of the Creative Commons Attribution 3.0 License, which permits unrestricted use, distribution, and reproduction in any medium, provided the original work is properly cited. 